\title{
Fabrication of High Performance Microlenses for an Integrated Capillary Channel Electrochromatograph with Flourescence Detection
}
J. R. Wendt, M. E. Warren, W. A. A. Allerman, T. R. Carter, R. E. Asbill, and S. Samora Sandia National Laboratories, Albuquerque, New Mexico 87185-0603

RECEIVED

We describe the microfabrication of an extremely compact optical system as a key element in an integrated capillary channel electrochromatograph with fluorescence detection. The optical system consists of a vertical cavity surface-emitting laser (VCSEL), two high performance microlenses and a commercial photodetector. The microlenses are multilevel diffractive optics patterned by electron beam lithography and etched by reactive ion etching in fused silica. The design uses substrate-mode propagation within the fused silica substrate. Two generations of optical subsystems are described. The first generation design has a $6 \mathrm{~mm}$ optical length and is integrated directly onto the capillary channelcontaining substrate. The second generation design separates the optical system onto its own substrate module and the optical path length is further compressed to $3.5 \mathrm{~mm}$. The first generation design has been tested using direct fluorescence detection with a $750 \mathrm{~nm}$ VCSEL pumping a $10^{-4} \mathrm{M}$ solution of CY-7 dye. The observed signal-to-noise ratio of better than 100:1 demonstrates that the background signal from scattered pump light is low despite the compact size of the optical system and is adequate for system sensitivity requirements.

\section{INTRODUCTION}

Integrated microsystems is an emerging technology in which electrical, optical, and mechanical functions are combined at the chip level into compact, lightweight, and [ultimately] low cost modules with performance equal to or even exceeding those of conventional macroscopic systems. Just as the invention of the integrated circuit revolutionized the electronics industry, the development of integrated microsystems is expected to revolutionize an even broader range of fields, extending beyond electro/optomechanical systems to the fields of biology, chemistry and medicine. And just as the integrated circuit combines formerly discrete devices (transistors, resistors, capacitors) onto a single chip with increasing functionality at decreasing cost, the integrated microsystem combines formerly separate subsystems (electronics, photonics, optics, fluidics, mechanics) onto a single multichip module. This results in significant size reduction and, hopefully, as the technology matures, to increased function with reduced cost.

One of the main areas of interest for the integrated microsystems work ongoing at Sandia National Laboratories (and around the world) is chemical detection and analysis for medical, industrial, and forensic applications.' Applications in such fields as hazardous waste 


\section{DISCLAIMER}

This report was prepared as an account of work sponsored by an agency of the United States Government. Neither the United States Government nor any agency thereof, nor any of their employees, make any warranty, express or implied, or assumes any legal liability or responsibility for the accuracy, completeness, or usefulness of any information, apparatus, product, or process disclosed, or represents that its use would not infringe privately owned rights. Reference herein to any specific commercial product, process, or service by trade name, trademark, manufacturer, or otherwise does not necessarily constitute or imply its endorsement, recommendation, or favoring by the United States Government or any agency thereof. The views and opinions of authors expressed herein do not necessarily state or reflect those of the United States Government or any agency thereof. 


\section{DISCLAIMER}

Portions of this document may be illegible in electronic image products. Images are produced from the best available original document. 
remediation, anti-terrorism, nonproliferation, and biotechnology are driving the development of miniaturized chemical analysis systems. Desirable attributes of such systems include small size, lightweight, low cost, and high sensitivity. These attributes lead to a system which can be handheld, deployed in the field in large numbers, and which will provide fast, accurate analysis previously requiring large, laboratory-based systems. A class of analytical techniques suitable for microsystem integration is electrokinetic capillary separation in conjunction with optical fluorescence detection. Electrokinetic capillary separations are traditionally performed in freestanding glass capillaries where a high voltage is applied across the fluid within the capillary to drive a sample through the capillary via electrokinetic flow. ${ }^{2}$ Different molecular components of the sample move at different rates through the capillary, the process known as electrophoresis, ${ }^{3}$ and, hence, become spatially separated. A laser pump and photodetector are placed near the end of the column and as the separated components pass by, a characteristic fluorescence signature can be obtained. In many cases, direct fluorescence from the chemical sample may be observed. In other cases, fluorescence is observed by tagging the chemical sample with an appropriate dye. Indirect fluorescence is an alternate detection method in which dye fluorescence is quenched by the presence of other chemicals.

Each of the components of a conventional capillary electrochromatograph exists in microscopic form and all may be fabricated by planar, lithographic-based techniques. The vertical cavity surface-emitting laser ${ }^{4}$ (VCSEL) can provide the pump beam. Multilevel binary lenses ${ }^{5}$ fabricated in fused silica can direct the pump beam and collect the fluorescence. Etched channels in any number of substrate materials (silicon and glass, ${ }^{6}$ quartz, ${ }^{7}$ plastic ${ }^{8}$ ) function the same as freestanding capillaries with the added advantages of requiring lower drive voltages, less sample volume, and reduced analysis time. The goal of this work is to combine these disparate elements into a compact module, often referred to as a lab-on-a-chip. The origin of this concept is attributed to a group at the Alberta Microelectronics Centre. ${ }^{9}$ The concept for the system described in this paper has been described by Warren. ${ }^{10}$ Similar work is being driven very strongly within the Human Genome Project where high-throughput DNA analysis is needed. ${ }^{11,12}$ 
Competing technologies for integrating pump light and capillary channels include fiber-based

pump sources, ${ }^{13}$ flow stream waveguides ${ }^{14}$ where light propagates down the channel itself, and conventional waveguides which are fabricated on the same substrate to intersect the capillary channel. ${ }^{15}$ The keys to the compact design of the Sandia system are the use of substrate-mode propagation for the optical path and high performance diffractive microlenses for coupling the pump beam into the substrate mode and for collecting and collimating the fluorescence from the capillary channel. In this work, we describe the design of two generations of optical subsystems, the microfabrication of the microlenses critical to the optical systems, and initial results from a complete integrated capillary channel electrochromatograph using direct fluorescence detection of a dye.

\section{DESIGN AND FABRICATION}

Two generations of optical systems have been designed and fabricated in this work. The first generation design is shown in Fig. 1. In this design, the microoptics are fabricated onto the same fused silica substrate that contains the capillary channel. This has the advantage of requiring no alignment or assembly steps for the optical system, exclusive of the VCSEL and photodetector. The VCSEL is flip-chip bonded onto a second fused silica cover plate and the photodetector, fronted by an interference filter, is mounted on the electronics subassembly. Because the fluorescence signal is only $30 \mathrm{~nm}$ longer in wavelength than the VCSEL pump beam, the high performance interference filter is necessary to block scattered pump light. Appropriate alignment marks and a custom precision die attach system provide for accurate alignment of the various subassemblies. The two microlenses are designed for a wavelength of $750 \mathrm{~nm}$ and operate in reflection. The off-axis lens, with a diameter of $0.9 \mathrm{~mm}$, has a deflection angle of $45^{\circ}$ to couple the VCSEL output into the substrate mode. The on-axis lens, with a diameter of $2 \mathrm{~mm}$, is an annular design that collects the fluorescence and directs it to the photodetector. This lens is designed to collimate the fluorescence so that the interference filter will efficiently transmit the fluorescence signal to the photodetector. The detector lens is very 
fast with a numerical aperture (NA) of 0.5 . Both lenses are computer designed Fresnel zone lenses, implemented in four layers (two etch steps). Details of the fabrication process are given below. Additionally, two metal mirrors serve to maintain the substrate-mode propagation over the $6 \mathrm{~mm}$ length of the optical system. A photograph of the first generation optical system is shown in Fig. 2.

The second generation design is shown in Fig. 3. In this design, the microoptics are fabricated on a separate fused silica substrate from the capillary channel. This modular design allows for more efficient fabrication of the optical subassembly, in parallel with fabrication of the capillary channels. The length of the optical system has also been reduced by almost a factor of two from the first generation design. Alignment of the various subassemblies is accomplished similarly to that for the first generation. The two microlenses are designed for a wavelength of $750 \mathrm{~nm}$ but now operate in transmission. The off-axis lens, with a diameter of $0.5 \mathrm{~mm}$, has a deflection angle of 26.5 to couple the VCSEL output into the substrate mode. The on-axis lens, with a diameter of $2.8 \mathrm{~mm}$, collects and collimates the fluorescence to the photodetector. The detector lens is again an aggressive design with high NA. As above, both lenses are computer designed Fresnel zone lenses, implemented in four layers (two etch steps). The fabrication process is very similar to that used for the first generation design but the differences are described below. Also as above, two metal mirrors serve to maintain the substrate-mode propagation over the shortened $3.5 \mathrm{~mm}$ length of the optical system. A photograph of the second generation optical system is shown in Fig. 4.

Fabrication begins with conventional optical lithography and standard semiconductor processing to define three sets of $\mathrm{Cr} / \mathrm{Au}$ alignment marks on the optically flat, two inch diameter fused silica substrate. The different sets of alignment marks are used for electron beam lithography, dual-side optical lithography, and for assembly of the subassemblies.

The electron beam lithography is performed on a JEOL JBX-5FE thermal field emission system operating at $50 \mathrm{kV}$. Lithographic challenges of the microlenses include submicron features $\left(0.15 \mu \mathrm{m}\right.$ minimum lines and spaces), relatively large areas $\left(1-3 \mathrm{~mm}^{2}\right)$, and an insulating 
substrate (fused silica). These challenges are met by utilizing optimized electron beam lithography processes described below. While this is not a low-cost technique, it is the most efficient and productive during the development phase. Once a design of the optical subassembly is finalized, it should be possible to substitute a fabrication technique for the lenses or for the entire optical subassembly more suitable for low cost, mass production. Possible techniques include plastic injection molding ${ }^{16}$ or embossing. ${ }^{17}$

The process sequence for microlens fabrication in the first generation design is now described. The beam current used is $2 \mathrm{nA}$ with a corresponding beam diameter of approximately $12 \mathrm{~nm}$. The addressed pixel spacing is $10 \mathrm{~nm}$ in an $80 \mu \mathrm{m}$ field. The resist used is polymethylmethacrylate (PMMA) at a thickness of $300 \mathrm{~nm}$ for the first level (the finer level) and $400 \mathrm{~nm}$ for the second level (the coarser level). The resist thickness for the second level is increased to provide better step coverage on the first level etched features. Prior to exposure, the PMMA is coated with $7.5 \mathrm{~nm}$ of thermally deposited Au for charge control, necessary because of the insulating nature of the fused silica. Both lens levels receive a dose of $425 \mu \mathrm{C} / \mathrm{cm}^{2}$. Prior to development, the thin Au layer is removed in a solution of potassium iodide and iodine. The PMMA is developed for 70 seconds in a 1:3 solution of methyl isobutyl ketone and isopropyl alcohol. The first level etch mask consists of $5 \mathrm{~nm} \mathrm{Cr} / 50 \mathrm{~nm} \mathrm{Ni}$ (the $\mathrm{Ni}$ thickness is increased to $75 \mathrm{~nm}$ for the second level etch) deposited by electron beam evaporation followed by liftoff in acetone. Etching is performed using reactive-ion etching with a $\mathrm{CHF}_{3} / \mathrm{O}_{2}$ chemistry. Desired etch depths are achieved by timed etches based on system calibration. Typical etch times are in the range of 5-10 minutes. After completion of the second level etch and removal of the Ni etch mask, the microlenses are coated with $150 \mathrm{~nm}$ of Au so that the lenses operate in reflection.

The process sequence for microlens fabrication in the second generation design is analogous to that for the first generation with various refinements. The beam current is increased to $5 \mathrm{nA}$ and the addressed pixel spacing is increased to $15 \mathrm{~nm}$ in an $80 \mu \mathrm{m}$ field, to reduce the exposure time on the large detector lens. The PMMA resist thickness is reduced to $200 \mathrm{~nm}$ for the first level to increase the process latitude. At the completion of etching, these lenses are not 
coated with Au because they operate in transmission.

\section{EXPERIMENTAL RESULTS}

Characterization of the first generation optical subassembly began by testing the module independently of the other subassemblies to confirm that the optical design performed as expected. First, the output characteristic of a flip-chip mounted VCSEL was measured. Then, that same VCSEL was mounted onto the fused silica substrate containing the optical system. By coupling the laser pump beam from the substrate (where it would normally be incident on the channel) with an index-matched $45^{\circ}$ prism, the pump beam power after transmission through the optical system was measured. The overall efficiency of the excitation part of the optical system is estimated to be at least $37 \%$. While the maximum possible efficiency of a [low NA, or slow] four-level binary optic is $81 \%$, the very fast detector lens used in this work will have a lesser maximum efficiency even before accounting for imperfections in fabrication. For the purposes of this work, the achieved efficiency is deemed adequate.

The complete first generation system, including VCSEL, channel, and photodetector, has been tested using fluorescence detection with a $750 \mathrm{~nm}$ VCSEL pumping a dilute solution of CY-7 dye. Collected fluorescence was measured first with only the buffer solution in the channel. Then a $10^{-4} \mathrm{M}$ solution of $\mathrm{CY}-7$ dye was introduced through the channel and the resulting fluorescence signal measured. The ratio of these two signal levels gives a signal-tonoise ratio of 100:1. This demonstrates that despite the compact size of the optical system, the background signal from scattered pump light is low. This analysis system is ultimately designed for indirect fluorescence detection of explosives and related degradation products. Experiments with explosive related chemicals using conventional capillary tubing have shown that a $10^{-5} \mathrm{M}$ solution of $\mathrm{CY}-7$ dye will be adequate. An open channel separation of $\mathrm{CY}-7$ was also performed with the first generation system. A three-peak fluorescence signature corresponding to the three molecular components of $\mathrm{CY}-7$ was collected in three minutes. 
Development of the capillary channels has been proceeding independently of the optical system. Electrophoretic separations and indirect fluorescence detection have been performed in fused silica channels on a sample containing eleven explosives and degradation products. These separations utilized an off-chip pump and photodetector setup. Nine of the eleven chemical constituents were identified in under one minute. ${ }^{18}$ It remains to perform a separation and analysis of a real sample on the fully integrated system. This will most likely be performed using the second generation optical subassembly, the assembly and test of which is in progress.

\section{SUMMARY}

We have described a highly integrated miniature chemical analysis system in the form of a compact capillary channel electrochromatograph with fluorescence detection. The use of high performance binary microlenses enables achievement of extremely compact and adequately efficient optical detection subassemblies. The use of microfabrication techniques for the bulk of the optical system provides inherent alignment and offers the possibility for economical mass production. Alignment of the subassemblies for assembly into an integrated microsystem is performed on a custom die attach apparatus, making use of lithographic alignment marks included on each subassembly. Tests of the first generation design using fluorescence detection with a $750 \mathrm{~nm}$ VCSEL pumping a $10^{-4} \mathrm{M}$ solution of CY-7 dye show a signal-to-noise ratio of better than 100:1, demonstrating that the background signal from scattered pump light is low despite the compact size of the optical system. An open channel separation of CY-7 dye has also been performed, further demonstrating the functionality of the overall system. The successful demonstration of two generations of optical subassemblies and the incorporation of the first generation design into a functional miniaturized chemical analysis system is a significant step forward along the path to an affordable, hand held chemical sensor. 


\section{ACKNOWLEDGMENT}

Sandia is a multiprogram laboratory operated by Sandia Corporation, a Lockheed Martin Company, for the United States Department of Energy under Contract DE-AC04-94AL85000. 


\section{FIGURE CAPTIONS}

Figure 1. Schematic cross section along the optical path of the first generation chemical analysis system. The off-axis lens couples the VCSEL pump beam into the substrate mode and two mirrors direct the beam to the capillary channel. The annular collection lens collimates the fluorescence to the detector. Both diffractive optics are coated with Au and operate in reflection.

Figure 2. Photograph of the first generation diffractive optical system, viewed from an oblique angle. Visible from left to right are the off-axis lens (dark oval), the two metal mirrors (bright ovals), and the collection lens (annulus). The capillary channel is not shown.

Figure 3. Schematic cross section along the optical path of the second generation chemical analysis system. The optical subassembly is now independent of the capillary channel, and the diffractive lenses operate in transmission.

Figure 4. Photograph of the second generation diffractive optical system, viewed normal to the surface. Visible from left to right are the off-axis lens (small light circle), the two metal mirrors (black circles), and the collection lens (large notched circle). 


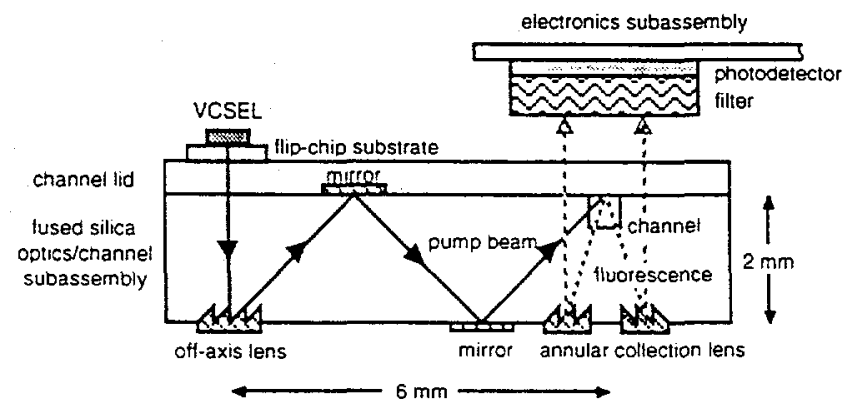

Figure 1. Schematic cross section along the optical path of the first generation chemical analysis system. The off-axis lens couples the VCSEL pump beam into the substrate mode and two mirrors direct the beam to the capillary channel. The annular collection lens collimates the fluorescence to the detector. Both diffractive optics are coated with Au and operate in reflection. 


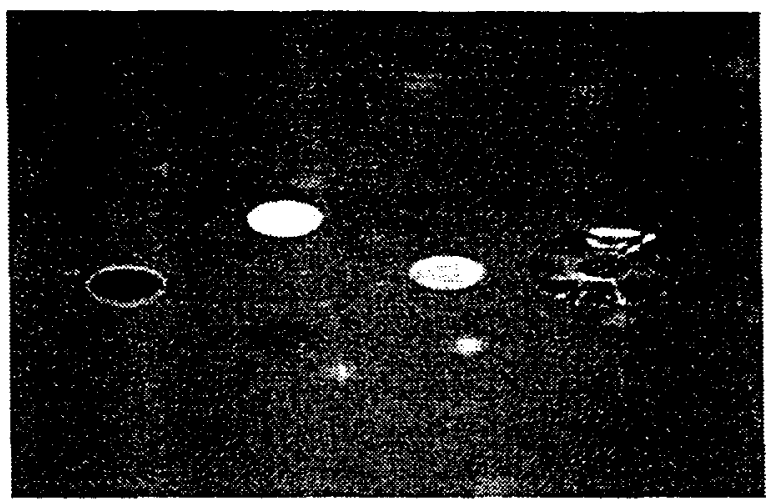

Figure 2. Photograph of the first generation diffractive optical system, viewed from an oblique angle. Visible from left to right are the off-axis lens (dark oval), the two metal mirrors (bright ovals), and the collection lens (annulus). The capillary channel is not shown. 


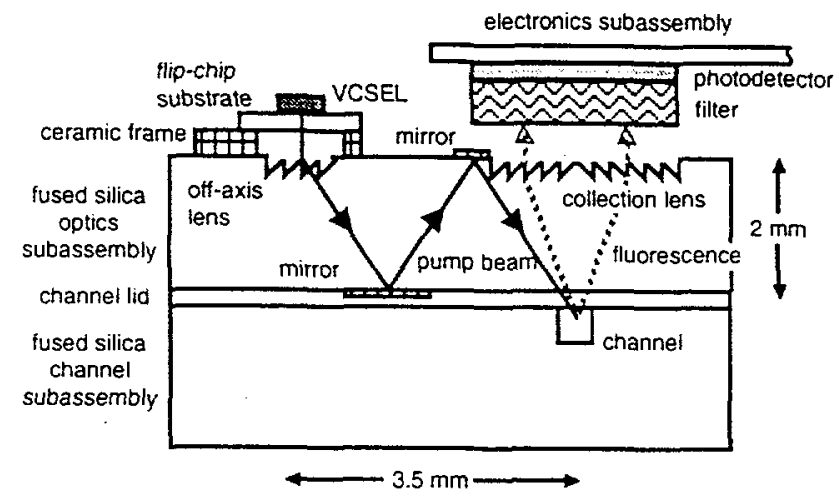

Figure 3. Schematic cross section along the optical path of the second generation chemical analysis system. The optical subassembly is now independent of the capillary channel, and the diffractive lenses operate in transmission. 


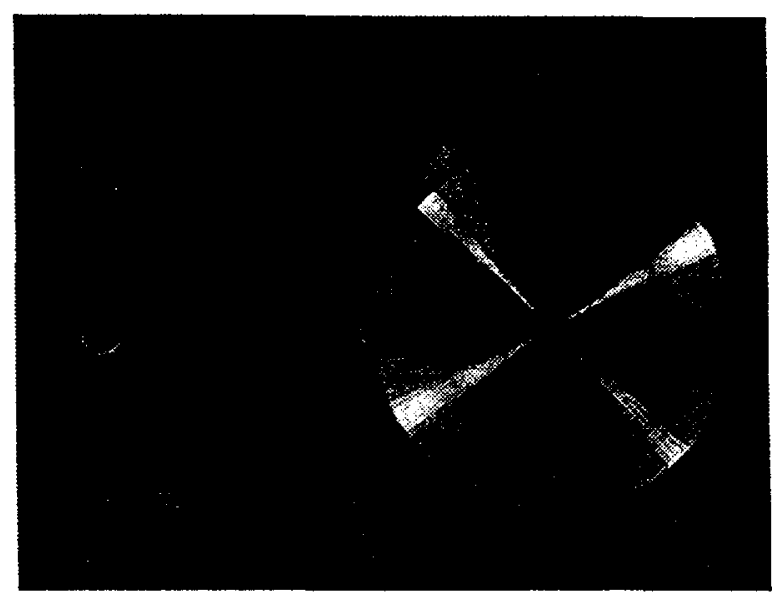

Figure 4. Photograph of the second generation diffractive optical system, viewed normal to the surface. Visible from left to right are the off-axis lens (small light circle), the two metal mirrors (black circles), and the collection lens (large notched circle). 
'For a broad range of recent work in this field see Proc. $\mu$-TAS '98. Banff (1998).

'M. F. M. Tavares and V. L. McGuffin, Anal. Chem. 67. 3687 (1997).

'C. Yan. R. Dadoo. R. N. Zare. D. J. Rakestraw, and D. S. Anex, Anal. Chem. 68. 2726 (1996).

‘ K. L. Lear. K. D. Choquette. R. P. Schneider. S. P. Kilcoyne, and K. M. Geib. Electron. Lett. 31. 208 (1995).

${ }^{5}$ G. J. Swanson and W. B. Veldkamp. Opt. Eng (Bellingham) 28, 605 (1989).

"A. Manz. J. C. Fettinger. E. Verpoorte. H. Lundi, H. M. Widmer, and D. J. Harrsion, Trends in Anal. Chem. 10, $144(1991)$.

'C. M. Matzke. D. W. Arnold, C. I. H. Ashby. S. H. Kravitz, M. E. Warren, and C. G. Bailey, Proc. SPIE 3515, 164 (1998).

${ }^{\rtimes}$ P. M. Martin. D. W. Matson, W. D. Bennett, and D. J. Hammerstrom, Proc. SPIE 3515, 172 (1998).

"A. Manz. D. Harrison, E. Verpoote, J. Fettinger. A. Paulus, H. Luedi. and H. Widmer, J. Chromatography 593, 253 (1992).

1" M. E. Warren. R. F. Carson, W. C. Sweatt. J. R. Wendt, J. A. Nevers. M. Hagerott Crawford. and H. Q. Hou. Proc. SPIE 3286, 42 (1998).

"A. T. Woolley, K. Lao, A. N. Glazer, and R. A. Mathies. Anal. Chem., pp (1998).

12 S. N. Brahmasandra, B. N. Johnson, J. R. Webster, D. T. Burke, C. H. Mastrangelo, and M. A. Burns. Proc. SPIE 3515. 242 ( 1998 ).

15 K. D. Kramer. K. W. Oh, C. H. Ahn, J. J. Bao. and K. R. Wehmeyer, Proc. SPIE 3515, 76 (1998).

${ }^{14}$ R. P. Mariella Jr.. G. van den Engh, D. Masquelier, and G. Eveleth, Cytometry 24, 27 (1996).

${ }^{15}$ M. E. Foquet. J. Han, A. Lopez. W. Wright, and H. G. Craighead, Proc. SPIE 3258, 141 (1998).

${ }^{16}$ R. M. McCormick. R. J. Nelson, M. G. Alonso-Amigo. D. J. Benvegnu, and H. H. Hooper, Anal. Chem. 69, 2626 (1997).

${ }^{17}$ J. Schulze. W. Ehrfeld. H. Müller, and A. Picard, Proc. SPIE 3289, 22 (1998).

${ }^{13}$ C. G. Bailey. et al., in preparation. 